\title{
Bistatic extension for coherent MMW-ISAR-Imaging of objects and humans
}

\author{
S. Bertl, A. Dallinger, and J. Detlefsen
}

Technische Universität München, Fachgebiet Hochfrequente Felder und Schaltungen, Arcisstr. 21, 80333 München, Germany

\begin{abstract}
We present a bistatic extension of a broadband monostatic FMCW Radar working in the Millimetre-Wave (MMW) region and its bistatic imaging properties used for imaging purposes. Due to the different perspective of a bistatic setup compared to a monostatic one, additional information can be obtained.

A wide bandwidth of approx. $10 \mathrm{GHz}$ is used for the task of high resolution imaging as it could be used for the detection of threats at a person's body in security-sensitive environments. Since MMWs propagate easily through common clothing, it is feasible to image objects like concealed weapons worn under the clothing. MMW-Imaging of humans is one possibility to enhance the capabilities of nowadays security checkpoints, e.g. at airports.
\end{abstract}

\section{Introduction}

For detection of concealed objects on the body of a person, different perspectives have to be applied in order to avoid shadowing effects. Therefore a setup with two sensors, one above and one below the person, is used as shown in Fig. 1. Both sensors are equipped with two receiving channels slightly displaced from each other. This allows the evaluation of the interferometric phase in order to obtain a threedimensional image. This aspect is covered in Bertl et al. (2007) and is not considered in the following. The original setup is a purely monostatic one. The two sensors are moved on a circular trajectory all around the person. The image is reconstructed by means of the Synthetic Aperture Radar (SAR) principle.

By combining the two sensors coherently, both the monostatic signal and the signal of the other sensor can be pro-

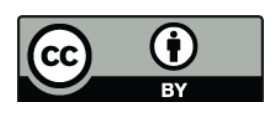

Correspondence to: $\mathrm{S}$. Bertl

(sebastian.bertl@tum.de) cessed. In the following the properties of this bistatic signals and the resulting images will be discussed. Also a realisation of a bistatic extension will be presented. Since the resolution capabilities of a bistatic setup differ from that of the monostatic setup, additional information can be obtained by such an extension.

\section{Bistatic imaging properties}

\subsection{Reconstruction algorithm}

In order to reconstruct calibrated bistatic signals an adapted backprojection algorithm can be used. The location of the reconstructed point $\boldsymbol{r}_{\mathrm{o}}$ and aperture coordinates $\boldsymbol{r}_{\mathrm{a}}$ are given by

$\boldsymbol{r}_{\mathrm{o}, \mathrm{a}}=(x, y, z)_{\mathrm{o}, \mathrm{a}}=\left(\rho_{\mathrm{o}, \mathrm{a}} \cdot \cos \varphi_{\mathrm{o}, \mathrm{a}}, \rho_{\mathrm{o}, \mathrm{a}} \cdot \sin \varphi_{\mathrm{o}, \mathrm{a}}, z_{\mathrm{o}, \mathrm{a}}\right)$.

The distance $R$ between the aperture position of the transmitter, the object position and the aperture position of the receiver then becomes $R=\left|\boldsymbol{r}_{\mathrm{a}, \mathrm{Tx}}-\boldsymbol{r}_{\mathrm{o}}\right|+\left|\boldsymbol{r}_{\mathrm{a}, \mathrm{Rx}}-\boldsymbol{r}_{\mathrm{o}}\right|$. The backprojected bistatic signal can be written as

$h\left(\boldsymbol{r}_{\mathrm{o}}\right)=\int_{\varphi_{\mathrm{a}, 1}}^{\varphi_{\mathrm{a}, 2}} u\left(R, \varphi_{\mathrm{a}}^{\prime}\right) \cdot \exp \left(j k_{\mathrm{c}} R\right) d \varphi_{\mathrm{a}}^{\prime}$.

\subsection{Bistatic resolution}

For the case of a point scatterer in the horizontal plane at the bisection of the two sensors, limits for the resolution will be derived in the following. This approach can be applied to arbitrary positions.

For the monostatic case the resolution in range becomes

$\Delta r_{\mathrm{ms}}=\frac{2 \pi}{\Delta k_{\mathrm{ms}}}=\frac{c_{0}}{2 B}$.

Published by Copernicus Publications on behalf of the URSI Landesausschuss in der Bundesrepublik Deutschland e.V. 


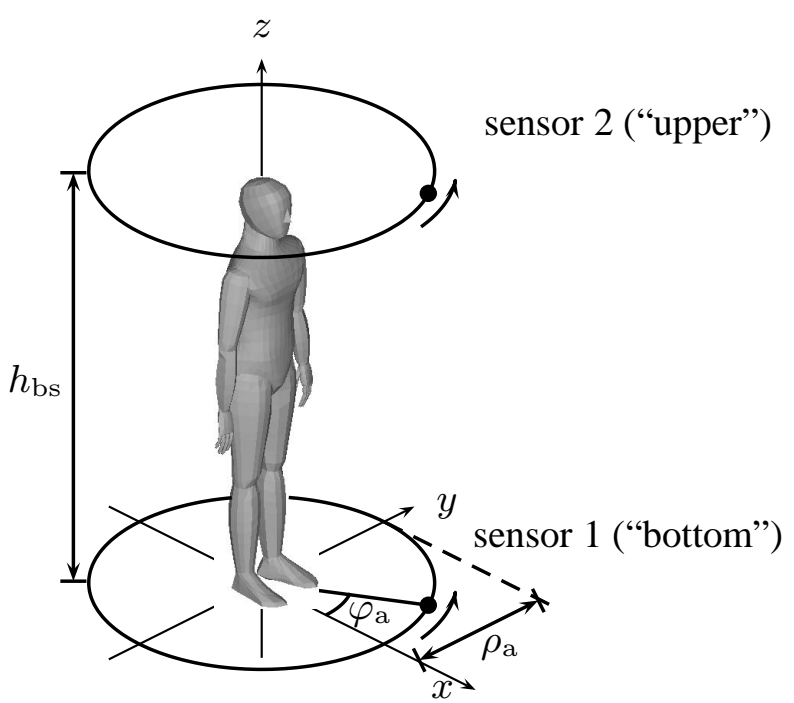

Fig. 1: Two sensors moving on a circular trajectory all around a person. The distance between sensor 1 and 2 is $h_{\mathrm{bs}}$.

where

$$
\begin{aligned}
\Delta k_{\mathrm{ms}} & =\left(k_{\mathrm{Tx}, \max }+k_{\mathrm{Rx}, \max }\right)-\left(k_{\mathrm{Tx}, \min }+k_{\mathrm{Rx}, \min }\right) \\
& =\frac{4 \pi}{c_{0}} \cdot\left(f_{\max }-f_{\min }\right)=\frac{4 \pi}{c_{0}} \cdot B .
\end{aligned}
$$

and $f \in\left[f_{\min }, f_{\max }\right]$. These considerations can be applied to the bistatic case as well. In contrast to the monostatic case, the $k$-vector has to be decomposed into in a $k_{\mathrm{x}}-$ and a $k_{\mathrm{Z}}$-component, depending on the position of the point under consideration for the Tx- and Rx-vector. For a point between the two sensors at a position $(x, y, z)=\left(\rho_{\mathrm{o}}, 0,0.5 \cdot h_{\mathrm{bs}}\right)$ this results in

$\boldsymbol{k}_{\mathrm{Tx}, \mathrm{bs}}=\left(k_{x}, k_{z}\right)=(\sin \alpha, \cos \alpha) \cdot k_{0}$

$\boldsymbol{k}_{\mathrm{Rx}, \mathrm{bs}}=\left(k_{x}, k_{z}\right)=(\sin \alpha,-\cos \alpha) \cdot k_{0}$

as shown in Fig. 2a. The resulting bistatic $k$-vector then becomes

$\boldsymbol{k}_{\mathrm{bs}}=\boldsymbol{k}_{\mathrm{Tx}, \mathrm{bs}}+\boldsymbol{k}_{\mathrm{Rx}, \mathrm{bs}}=(2 \sin \alpha, 0) \cdot k_{0}$.

By modulating the frequency by $\Delta f=f_{\max }-f_{\min }$ we obtain

$\Delta k_{\mathrm{bs}}=2 \sin \alpha \frac{2 \pi}{c_{0}}\left(f_{\max }-f_{\min }\right)=\sin \alpha \cdot \Delta k_{\mathrm{ms}}$

Taking the SAR-processing step into account, the radial bistatic resolution can be improved considerably. The relevant angle of observation $\varphi_{\text {asp }}$ (see Fig. 2b) can be written in terms of the object location $\left(\rho_{\mathrm{o}}\right)$ and the aperture coordinates

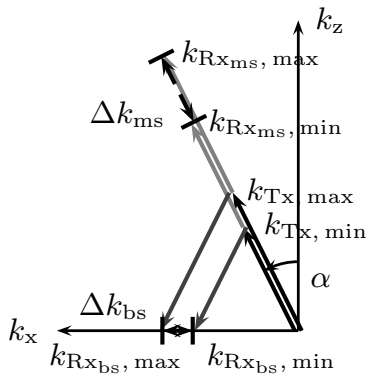

(a) mono- and bistatic $k$ vectors

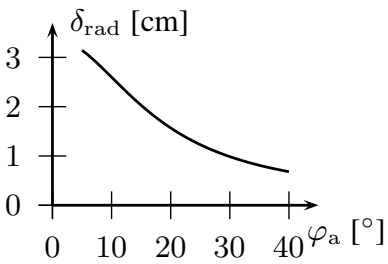

(c) radial resolution

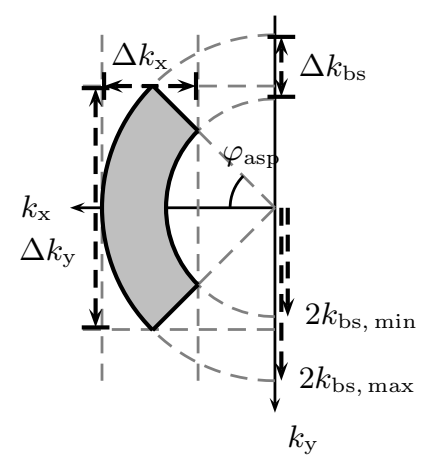

(b) bistatic $k$-space

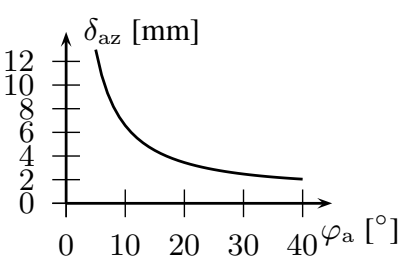

(d) horizontal resolution

Fig. 2: Bistatic resolution; Used parameters in (c) and (d): $f=90 \ldots 100 \mathrm{GHz}, \rho_{\mathrm{a}}=0.6 \mathrm{~m}, h_{\mathrm{bs}}=1.62 \mathrm{~m}, \rho_{\mathrm{o}}=0.2 \mathrm{~m}$, $z_{\mathrm{o}}=0.81 \mathrm{~m}$.

$\left(\rho_{\mathrm{a}}, \varphi_{\mathrm{a}}\right)$ as

$\tan \varphi_{\text {asp }}=\frac{\sin \varphi_{a} \cdot \rho_{a}}{\cos \varphi_{a} \cdot \rho_{a}-\rho_{o}}$,

where $\varphi_{a}$ is the aperture angle defined in Eq. (1). To determine the radial resolution capability, $\delta_{\mathrm{x}}$ respectively $\delta_{\mathrm{rad}}$, the width of the occupied $k$-space along this direction has to be taken into account. For the maximum and minimum we obtain

$2 k_{\max , \mathrm{bs}}=\frac{4 \pi f_{\max }}{c_{0}} \cdot \sin \alpha$

$2 k_{\min , \mathrm{bs}}=\frac{4 \pi f_{\min }}{c_{0}} \cdot \cos \varphi_{\mathrm{asp}} \cdot \sin \alpha$.

The radial resolution therefore becomes

$$
\begin{aligned}
\delta_{x}\left(=\delta_{\mathrm{rad}}\right) & =\frac{2 \pi}{\Delta k_{\mathrm{bs}, \mathrm{x}}}=\frac{2 \pi}{2 k_{\max , \mathrm{bs}}-2 k_{\mathrm{min}, \mathrm{bs}}} \\
& =\frac{c_{0}}{2\left(f_{\max }-f_{\min } \cdot \cos \varphi_{\mathrm{asp}}\right) \cdot \sin \alpha} .
\end{aligned}
$$

For the resolution along azimuth the same considerations lead to a resolution $\delta_{\mathrm{az}}$

$\delta_{\mathrm{y}}\left(=\delta_{\mathrm{az}}\right)=\frac{c_{0}}{4 f_{\max } \cdot \sin \alpha \cdot \sin \varphi_{\mathrm{asp}}}$.

With Eq. (10) the resolutions $\delta_{\text {rad }}$ and $\delta_{\mathrm{az}}$ can be written as a function of the aperture angle $\varphi_{\mathrm{a}}$. A plot of the two expressions for the resolution can be seen in Fig. $2 \mathrm{c}$ and $\mathrm{d}$. 


\section{Implementation of a bistatic measurement setup}

The proposed system setup uses one common voltage controlled oscillator (VCO) to combine the two originally monostatic sensors coherently. According to Fig. 3 one of the sensor signals is delayed in time by using a coaxial delay line. In addition to the monostatic signal, both sensors also receive the bistatic signal generated by the other.

The monostatic and the bistatic signals can be separated in the time domain because of a suitable choice of the length of the delay line. By adequate windowing the monostatic part can be extracted, and the further processing of the monostatic signal is done by common SAR algorithms as presented in Dallinger et al. (2006).

The SAR processing of the bistatic signal is done according to Eq. (2).

\subsection{Sensor description}

The FMCW-radar principle is used for each sensor. A schematic of the two sensors is given in Fig. 3a. A Voltage Controlled Oscillator (VCO) generates a frequencymodulated signal. The generated signal is multiplied to obtain an output-signal in the MMW-domain. A part of the Txsignal is transmitted by the Tx-antenna, the other part is used as the local oscillator (LO) signal. Since the homodyne detection principle is used, one MMW-source is sufficient. The received signal $(\mathrm{Rx})$ is fed to the mixer via a Low Noise Amplifier (LNA). The Rx-signal is shifted to baseband. Instead of an I/Q demodulator the analytic signal is calculated using the Hilbert transform. The frequency-sweep is done in $1-2 \mathrm{~ms}$. In conjunction with a circularly moving sensor a person can be measured within approx. $20 \mathrm{~s}$ over a full rotation of $360^{\circ}$. A more detailed description can be found in Detlefsen et al. (2006).

\subsection{Bistatic setup}

With the combined setup, given in Fig. 3a, mono- and bistatic measurements can be performed simultaneously.

Instead of two separately controlled signal sources only the source from (in this case) the bottom sensor is used. This signal is sent to the upper sensor via a coaxial delay line and is fed to the multiplier's input.

This means, that the same signal is transmitted by the bottom sensor first and then with a delay of $\Delta \tau_{12}$ by the upper sensor as well.

\subsection{Spectra of the difference frequencies}

The location of the resulting spectra observed at the two sensors is given in Fig. 3b, c. Black lines are related to Tx|Rxsignals generated by the bottom sensor, gray lines belong to the upper sensor. At the bottom sensor the monostatic part is received first. The delay $\tau_{\mathrm{ms}}$ of this measurement is proportional to the distance between transmitter $\mathrm{Tx}_{1}$, the scat-

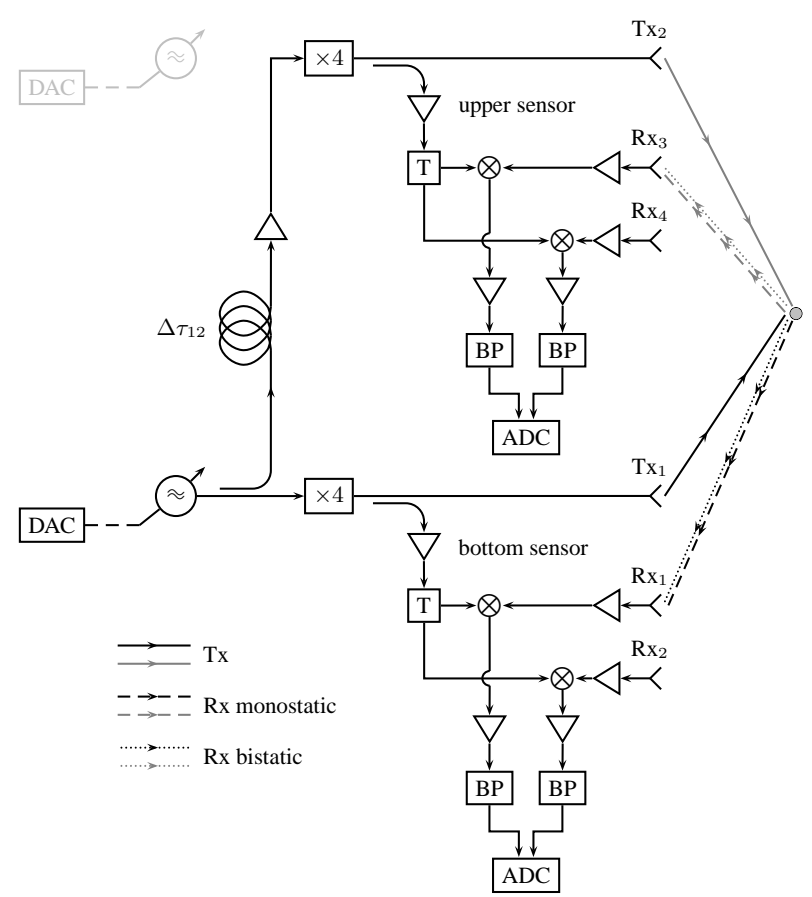

(a) System setup
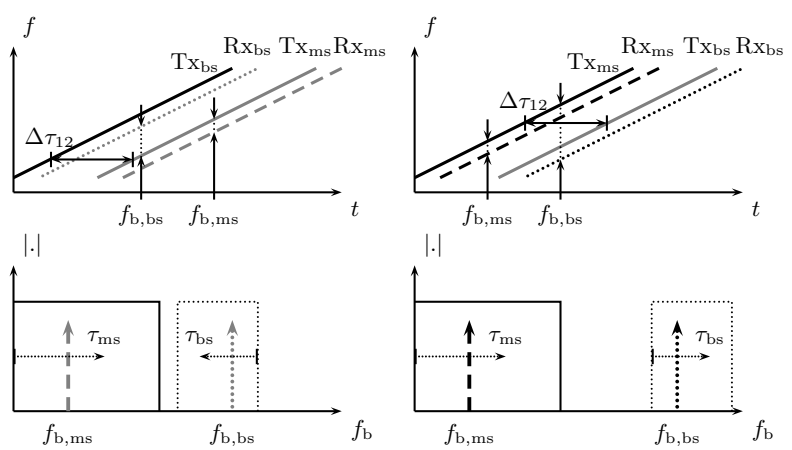

1.

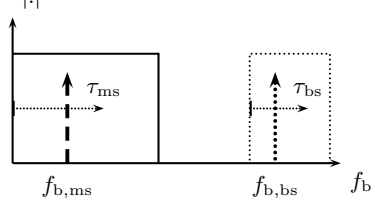

(b) Frequencyspectrum upper sen-(c) Frequencyspectrum bottom sor (Tx2, Rx3|4) sensor (Tx1, Rx1|2)

Fig. 3: Bistatic setup with two coherent (interferometric) Sensors for acquisition of mono- and bistatic measurement data.

tering center and the considered receiver $\mathrm{Rx}_{1 \mid 2}$. Here no changes compared to a purely monostatic measurement occur. The signal delayed by $\Delta \tau_{12}$, and transmitted from the upper sensor $\left(\mathrm{Tx}_{2}\right)$, is received directly by the bottom sensor or scattered at an object at first, which results in an additional travel time. After down-conversion using the Tx-signal (LOsignal) of the bottom sensor, the minimal beat-frequency for the bistatic part is obtained for the direct path. Scatterers at greater distances with respect to this direct path are represented by a larger beat frequency.

Considering the upper sensor, the chirp signal delayed by $\Delta \tau_{12}$ is radiated and used as LO-signal for the upper sensor. 


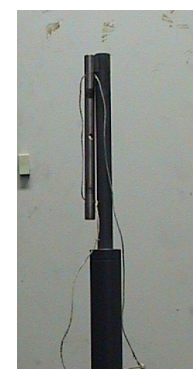

(a) Photo (side view)

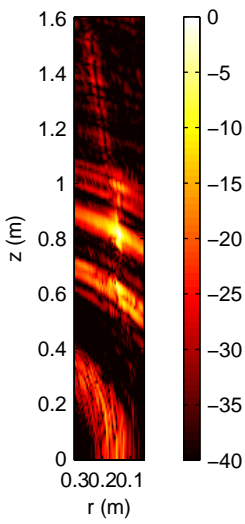

(c) monostatic (side view)

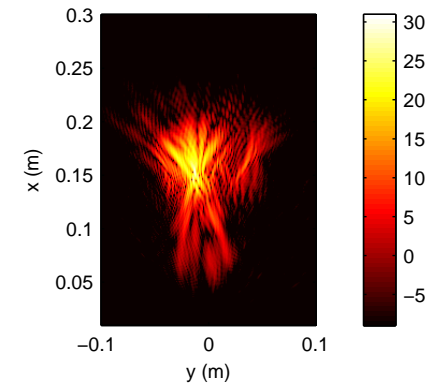

(b) Horizontal cut at $z_{\mathrm{o}}=$ $0.81 \mathrm{~m}$ (in $\mathrm{dB}$ )

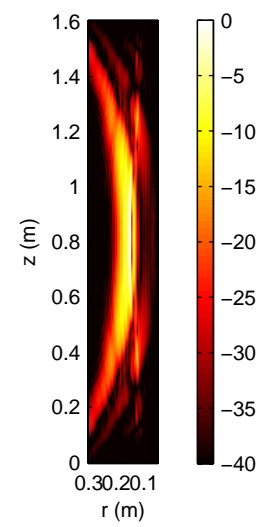

(d) bistatic (side view)

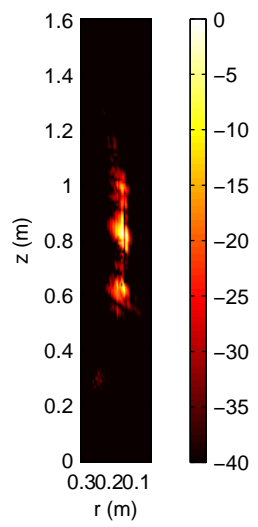

(e) incoherent mono- and bistatic combination
Fig. 4: Metallic rod mounted on a PVC-rod; aperture segment used for reconstruction $\varphi_{\mathrm{a}}= \pm 40^{\circ}$; Bandwidth $B=10 \mathrm{GHz} ; f=90 \ldots 101 \mathrm{GHz}$

Again for the monostatic signals at the upper sensor, no changes arise compared with a purely monostatic measurement.

For the bistatic signal the highest possible difference frequency with respect to the delayed reference signal is obtained by the direct crosstalk between sensor 1 and 2 . All responses from objects with a non-zero distance to this direct path will be mapped to a lower difference frequency. The spectrum of the bistatic component of the upper sensor is mirror-inverted, i.e. longer distances " $\mathrm{Tx}_{1}-$ scatterer $\mathrm{Rx}_{3 \mid 4}$ " are mapped to smaller difference frequencies as can be seen in Fig. 3c.

\subsection{Determination of the delay-line length}

In order to avoid overlapping of the spectra of the different signal components, several system parameters must be adjusted thoroughly. The maximum difference frequency occurs at the bistatic signal component of the bottom sensor.
The upper limit for the beat frequency can either result from the bandpass filter (denoted by "BP" in Fig. 3) or the available sampling rate. In this implementation the bandpass filter with a cut-off frequency of $300 \mathrm{kHz}$ is the limiting factor compared to the sampling rate of $1 \mathrm{MSample} / \mathrm{s}$. The lowest difference frequency appears for bistatic signals at the upper sensor. This frequency must not lie in the interval that is reserved for the monostatic signal components. The electrical length of the delay line was chosen to be $10.4 \mathrm{~m}$, which assures that the mono- and bistatic signals do not interfere and that the ambiguity range and the BP's cut-off frequency are not exceeded.

\section{Reconstruction results}

For testing purposes a vertically placed metallic rod with an approximate height of $20 \mathrm{~cm}$ and a diameter of $1 \mathrm{~cm}$ was used. This isolated object was used to test the properties of the bistatic images. The mono- and bistatic reconstruction results and their non-coherent combination can be seen in Fig. 4. The theoretically determined values for the horizontal resolution are met. The resolution in $z$-direction is, as expected, poor.

\section{Conclusions}

We presented an extension of two monostatic sensors up to a fully mono- and bistatic sensor network. In addition to the monostatic signals, the information of the bistatic signals can be evaluated too. The data processing and the quality of the reconstructed monostatic images is not affected. As a nice side-effect only one signal generator (VCO) is needed with this extension instead of two.

Calibration and reconstruction algorithms capable to handle bistatic data were developed. First reconstruction results for isolated objects show that the resolution which is achieved, is according to the theoretical values for this setup.

\section{References}

Bertl, S., Dallinger, A., and Detlefsen, J.: Broadband circular interferometric millimetre-wave ISAR for threat detection, Adv. Radio Sci., 5, 2007.

Cumming, I. G. and Wong, F. H.: Digital Processing of Synthetic Aperture Radar Data, Artech House, London, 2005.

Dallinger, A., Bertl, S., Schelkshorn, S., and Detlefsen, J.: SAR Techniques for the Imaging of Humans, in: EUSAR 2006, 6th European Conference on Synthetic Aperture Radar, Electronic Proceedings, VDE Verlag GMBH, Dresden, 2006.

Detlefsen, J., Dallinger, A., Schelkshorn, S., and Bertl, S.: UWB Millimeter-Wave FMCW Radar using Hilbert Transform Methods, pp. 46-48, Horizon House Publications, 2006.

Soumekh, M.: Fourier Array Imaging, Prentice Hall, Englewood Cliffs, N. J., 1994. 